\title{
Multiscale Coherence Regularization Reconstruction Using A Nonlocal Operator for Fast Variable-Density Spiral Imaging
}

Sheng Fang ${ }^{\mathrm{a}}$, Lyu Li ${ }^{\mathrm{b}}$,Wenchuan $\mathrm{Wu}^{\mathrm{b}}$, Juan Wei ${ }^{\mathrm{c}}$, Bida Zhang ${ }^{\mathrm{c}}$, Dong-Hyun Kim ${ }^{\mathrm{d}}$, Chun Yuan b,e and Hua Guo ${ }^{\text {b* }}$

${ }^{\mathrm{a}}$ Institute of Nuclear and New Energy Technology, Tsinghua University; ${ }^{\mathrm{b}}$ Center for Biomedical Imaging Research, Department of Biomedical Engineering, Tsinghua University, Beijing, China;

${ }^{\mathrm{c}}$ Philips Research Asia, Shanghai, China; ${ }^{\mathrm{d}}$ Department of Electrical and Electronic Engineering,

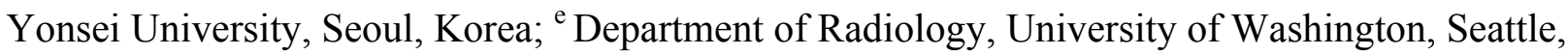
Washington, USA.

Word Count: 4138 (excluding this page)

*Correspondence to:

Hua Guo, $\mathrm{PhD}$

Center for Biomedical Imaging Research

Department of Biomedical Engineering

Tsinghua University, Beijing, China

Phone: +86-10-6279-5886

Email: huaguo@tsinghua.edu.cn

Grant sponsor: This work is supported by the National Natural Science Foundation of China, (11475100 and 61271132), and the Beijing Natural Science Foundation (7142091). 


\section{ABSTRACT}

Purpose: Nonlinear reconstruction can suppress pseudo-incoherent aliasing artifacts from variable-density spiral (VDS) trajectories when interleaves are undersampled for acquisition acceleration during MR imaging. However, large-scale aliasing artifact suppression often conflicts with fine-scale structure preservation and may cause deterioration of image quality in the reconstructed images. To address this issue, a sequential, multiscale coherence regularization algorithm using a nonlocal operator (mCORNOL) is proposed.

Methods: mCORNOL is formed by exploiting the scale-control capacity of nonlocal operators in image structure measurement. By changing the scale of the structure measurement, the smoothing constraint scales can be adjusted. Starting with a large value, mCORNOL gradually reduces the smoothing constraint scale until it reaches the same level as the noise. Therefore, the large-scale smoothing constraint dominates the first few iterations of the reconstruction and removes aliasing artifacts as well as fine structures. In the following iterations, the smoothing constraint is restricted to a smaller and smaller scale, so the fidelity term progressively dominates and restores lost structures. Thus, aliasing artifact removal and structure preservation can be decoupled and achieved sequentially, which alleviates the conflicts between them.

Results: Numerical simulation and in vivo experiment results demonstrate the superiority of mCORNOL for aliasing artifact suppression and image structure preservation at high reduction factors, compared to SENSE, Total Variation and the original CORNOL reconstruction.

Conclusions: mCORNOL reconstruction provides an effective way to improve image quality for undersampled VDS acquisitions. 
Key Words: variable-density spiral, parallel imaging, multiscale CORNOL, structure preservation, nonlinear reconstruction 


\section{Introduction}

The variable density spiral (VDS) [1,2] is an efficient data acquisition method that oversamples central k-space data while maintaining a fast k-space traversal speed. Since most of the energy in typical MR images is concentrated around the origin of k-space, oversampling of central k-space by VDS can provide benefits for various applications. For example, VDS can provide navigator information for motion artifact suppression in multishot high-resolution diffusion weighted imaging [3-7] and high-resolution coronary artery MRI [8]. VDS is also less susceptible to motion than other techniques and has been used in CINE imaging $[9,10]$.

In addition to central k-space oversampling, another beneficial feature of VDS is that its undersampling artifacts appear noise-like and thus incoherent. Previous studies have applied this feature to various applications, including MR fluoroscopy [11], cardiac imaging [1], perfusion imaging [12], and coronary MR angiography [13]. VDS has also recently received attention for application in both parallel imaging and compressed sensing (CS) MRI [14], due to the fact that incoherent aliasing artifacts can be suppressed without degrading the image quality, provided that an appropriate nonlinear reconstruction is constructed [15]. Potential candidates for such reconstruction include various nonlinear reconstruction methods that were previously explored for regularized parallel imaging [16-19] and CS reconstruction [14,20-24].

These nonlinear regularization algorithms generally rely on prior knowledge to exert adaptive smoothing constraints to suppress aliasing artifacts and image noise. Among them, coherence regularization with a nonlocal operator (CORNOL) is a recently proposed method that can smooth out pixel intensity oscillations for intra-structures while preserving inter-structure 
intensity changes by using a nonlocal operator [19]. Previous studies show CORNOL can remove incoherent aliasing artifacts effectively and retain image details for Cartesian cases. CORNOL has also been preliminarily combined with a VDS trajectory $[25,26]$.

For VDS acquisitions, the spatial scale of aliasing artifacts may have a large range of variance and display ringing artifacts due to the truncation of gridding kernels. Although small-scale artifact removal and fine structure preservation may be achieved simultaneously by imposing a small-scale smoothing constraint, this is usually difficult to achieve for large-scale artifacts. Large-scale artifact elimination requires large-scale smoothing constraints, and thus cannot be achieved simultaneously with fine structure preservation.

This contradiction may theoretically be solved using a multiscale reconstruction. Although there are some reports about multiscale L0 norm continuation [27] and multi-weight wavelet [28] reconstruction with remarkable results, these methods are not designed with the objective of solving the aforementioned contradiction. Therefore, it is necessary to develop a method that explicitly deals with artifact removal and structure preservation for VDS imaging.

For this purpose, a sequential multiscale CORNOL (mCORNOL) reconstruction was proposed in this study. It was formed by exploiting the scale-control capacity of nonlocal operators in image structure measurement. Instead of performing a "one-pass" reconstruction with a fixed smoothing scale, mCORNOL iteratively performs CORNOL while reducing the scales of the imposed smoothing constraint every few iterations. With large scale smoothing in the first half of the reconstruction, the smoothing effect dominates and removes aliasing artifacts as well as fine structures. As the iteration proceeds, the scale of the smoothing constraint 
gradually decreases to the scale of noise, so that the fidelity term dominates. By enforcing data consistency, the fidelity term restores the previously lost structures. As such, large-scale artifact suppression and fine-scale structure preservation can be decoupled and performed sequentially, and the conflicts between them can be alleviated.

Except simulation, the proposed mCORNOL was also tested with in vivo data acquired by a fast VDS acquisition technique, making it closer to practical use than previous studies. mCORNOL was compared with non-regularized SENSE, Total Variation (TV) [29], and CORNOL. The results demonstrate that the proposed method outperforms the other three methods in both large-scale artifact removal (e.g. aliasing artifacts and ringing artifacts) and fine-scale image structure preservation.

\section{Materials and Methods}

\subsection{Constrained reconstruction}

For an acquisition using phased array RF coils, the imaging process can be formulated as a linear operation using the generalized SENSE model [30]:

$$
\overline{\mathbf{f}}=\mathbf{A} \overline{\mathbf{u}}+\sigma
$$

where $\overline{\mathbf{f}}$ is a vector formed by the k-space data acquired from all receiver coils, $\overline{\mathbf{u}}$ is the unknown vector describing the desired image and $\sigma$ is the noise. The system matrix $\mathbf{A}$ consists of the product of the undersampled trajectory, Fourier encoding, and coil sensitivity. In

non-Cartesian SENSE reconstruction, the desired image $\overline{\mathbf{u}}$ is usually estimated using iterative numerical schemes, such as the linear conjugate gradient method [30] with given values for $\mathbf{A}$ and $\overline{\mathbf{f}}$. 
An issue with the linear reconstruction is that the system matrix $\mathbf{A}$ could be poorly conditioned for certain coil geometries at high reduction factors. In these cases, regularization is needed to constrain the reconstruction, which can be formulated as:

$$
\overline{\mathbf{u}}^{\mu}=\underset{\bar{u}}{\arg \min }\left\{\|\mathbf{A} \overline{\mathbf{u}}-\overline{\mathbf{f}}\|_{2}^{2}+\mu J(\overline{\mathbf{u}})\right\}
$$

Here \|\|$_{2}$ is the $L_{2}$ norm. $\|\mathbf{A} \overline{\mathbf{u}}-\overline{\mathbf{f}}\|_{2}^{2}$ is the fidelity term that unfolds aliasing and reveals true image structures by enforcing the consistency with acquired data and the SENSE model. If $\mathbf{A}$ is ill-conditioned, it may introduce amplified noise or residual aliasing artifacts. $J(\overline{\mathbf{u}})$ is the regularization term. It can suppress noise and possibly remove residual aliasing artifacts (for some nonlinear regularization methods) by promoting some desirable properties in the solution based on prior information. However, it may also degrade fine structures, if the prior information is not appropriate. Thus the effects of these two terms, fidelity and regularization, conflict with each other. $\mu$ is the regularization parameter which creates a trade-off between them.

\subsection{Image scales and CORNOL}

A key source of this conflict is the scales of image components, including residual aliasing artifacts, image structure and noise. Here, the scale means the spatial range of these components. For white noise, its spatial scale or extent can be the size of a single spatial sample, i.e. one voxel. If the noise is correlated, the scale of noise may be the size of several single spatial samples or even more. For residual aliasing artifact, its size can be a portion of a whole image. If the scale of a residual aliasing artifact is smaller than that of a fine structure, a single scale smoothing constraint can remove residual aliasing artifacts without degrading the image structures. 
Otherwise, the conflict between residual aliasing artifact removal and fine structure preservation can cause the image structure to degrade, which often happens in practice. In this case, a single scale smoothing constraint may fail to meet these two demands.

An intuitive solution to alleviate the conflict that arises from handling multiscale components in the aliased images is to iterate Eq. (2) while changing the scales of the smoothing constraints at every iteration. Each iteration then focuses on handling image components of a single scale and the aforementioned conflict can be alleviated.

In this study, the CORNOL method was chosen as the base of such a sequential multiscale reconstruction method, since it has an explicit scale control parameter. Its regularization term can be expressed by the inner product of a structure-adaptive weight vector and an intensity difference based vector [19]:

$$
J_{\text {CORNOL }}(\overline{\mathbf{u}})=\sum_{i \in \Omega}\left\langle\phi_{i}^{\mathrm{r}}, \nabla u_{i}^{\mathrm{r}_{\mathrm{o}_{2}}}\right\rangle
$$

Here $\nabla \stackrel{1}{u_{i}}$ is a vector of intensity differences between pixel $i$ and certain selected pixels. The notation ${ }^{\circ} 2$ denotes element-wise square. $\stackrel{1}{\phi}_{i}$ is a vector comprising weights for each elements of $\nabla \stackrel{1}{u_{i}}$, which is defined as:

$$
\begin{gathered}
\stackrel{\mathrm{r}}{\phi_{i}}=\left\{\phi_{i j}\right\}, \phi_{i j}=\frac{1}{W_{i}} \exp \left(-\frac{\|p(i)-p(j)\|_{2}^{2}}{h^{2}}\right), j \in N(i) \\
W_{i}=\sum_{j \in N(i)} \phi_{i j}
\end{gathered}
$$

where $\phi_{i j}$ is the weight that quantifies the relevance between pixels $i$ and $j$; the notation $j \in N(i)$ denotes pixel $j$ being in a set selected for pixel $i$, which is formed by pixels in a square search window centered at pixel $i ; p(i)$ and $p(j)$ are two patches centered at pixel $i$ and pixel $j$, which 
are two subsets of the desired image $\overline{\mathbf{u}} ; W_{i}$ is a normalization factor that sums up all weights for pixel $i$; $h$ is a threshold parameter which controls the shape of exponential decay and determines how many pixels are involved in the smoothing constraint. Therefore, it explicitly controls the scale of smoothing constraint in CORNOL. Given $h$, the scale of smoothing constraint is fixed and the weight vector in Eq. (4) can distinguish intra-structure intensity oscillations from inter-structure intensity changes based on this scale, such that minimization of Eq. (3) can adaptively suppress inter-structure intensity changes, which primarily result from noise or small scale residual aliasing artifacts.

Fig. 1 demonstrates the scale selection effect of the nonlocal operators, by computing Eq. (4) with different threshold parameters. The top row compares the shape of the exponential term in Eq. (4), which dominates the magnitude of weights for each pixel. For pixels whose patch difference $\|p(i)-p(j)\|_{2}^{2}$ is large, the exponential decay filters them out by setting its weight close to zero, so as to avoid the inappropriate smoothing among different image structures. As $h$ decreases, the decay of the exponential term becomes sharper, so that the selection based on patch difference becomes more exclusive and fewer pixels are involved in regularization. The bottom row shows the effect of $h$ on the computation of weights $\phi_{i j}$. When $h$ is large, the weight can not identify local structures exactly (Fig. 1d). With a moderate $h$, the weight can detect local edges effectively (Fig. 1e). For an even smaller $h$, the weights are further restricted to edges (Fig. 1f).

\subsection{Multiscale CORNOL}


Based on CORNOL, the proposed sequential multiscale CORNOL (mCORNOL) can be formulated as:

$$
\begin{gathered}
\overline{\mathbf{u}}_{k}^{\mu}=\underset{\overline{\mathbf{u}}}{\arg \min }\left\{\|\mathbf{A} \overline{\mathbf{u}}-\overline{\mathbf{f}}\|_{2}^{2}+\mu J_{\text {CORNOL }}^{h_{k}}(\overline{\mathbf{u}})\right\} \\
h_{k}=\alpha^{-k} h_{0}
\end{gathered}
$$

where $k$ is the number of iterations; $h_{k}$ is the threshold parameter for the $k$-th iteration; $h_{0}$ defines the initial threshold parameter and $\alpha$ is a tuning factor that changes the scale parameter.

In this study, $\alpha$ is set to 2 for all reconstructions, which means that the scale of the smoothing constraint is decreasing during reconstruction. At the beginning of the reconstruction, a large smoothing constraint is applied in order to remove aliasing artifacts. Meanwhile, fine image structures will also be removed. As the iteration proceeds, the scale of smoothing constraint decreases, which consecutively reduces the spatial range of the smoothing constraint. Note that the result of previous iteration is inherited as an initial guess for the present in each iteration step. The regularization term $\mu J_{\text {CORNOL }}^{h_{k}}(\overline{\mathbf{u}})$ is gradually limited to an increasingly subtle

spatial scale and the fidelity term $\|\mathbf{A} \overline{\mathbf{u}}-\overline{\mathbf{f}}\|_{2}^{2}$ progressively dominates Eq. (6). Through the unfolding effect by the fidelity term, aliasing artifacts can be suppressed as the scale of smoothing constraint decreases. The fidelity term also enforces the consistency between the resultant image and acquired data, so previously removed structures are gradually recovered, as long as the fidelity term prevails over the regularization term.

\subsection{Implementation}

The proximal forward-backward splitting algorithm [31] used for the original CORNOL was modified to implement the proposed mCORNOL in this study. The specific algorithm can be 
described using the following pseudo code:

$$
\begin{aligned}
& \overline{\mathbf{u}}_{0}=0, \sigma_{0}=\left\|\mathbf{A} \overline{\mathbf{u}}_{0}-\overline{\mathbf{f}}\right\|_{2}, h_{k}=h_{0} \\
& \text { do } \\
& \overline{\mathbf{z}}_{k}=\overline{\mathbf{u}}_{k-1}-\frac{1}{\eta} \mathbf{A}^{H}\left(\mathbf{A} \overline{\mathbf{u}}_{k-1}-\overline{\mathbf{f}}\right) \\
& \text { compute weights } \stackrel{\mathrm{r}}{\phi_{i}} \text { based on } \overline{\mathbf{z}}_{k} \text { and } h_{k} \\
& \overline{\mathbf{u}}_{k}=\underset{\overline{\mathbf{u}}}{\arg \min }\left\{\left\|\overline{\mathbf{u}}-\overline{\mathbf{z}}_{k}\right\|_{2}^{2}+\mu \eta \sum_{i \in \Omega}\left\langle\stackrel{\mathrm{r}}{\phi_{i}}, \nabla r_{i}^{\mathrm{r}_{{ }^{2}}}\right\rangle\right\} \\
& \text { if }\left(\frac{\left\|\overline{\mathbf{u}}_{k}-\overline{\mathbf{u}}_{k-1}\right\|_{2}}{\left\|\overline{\mathbf{u}}_{k-1}\right\|_{2}}<\tau_{b} \text { and } h_{k}>h_{\text {lowbound }}\right) \quad h_{k}=\alpha^{-k} h_{0} \\
& \sigma_{k}=\left\|\mathbf{A} \overline{\mathbf{u}}_{k}-\overline{\mathbf{f}}\right\|_{2} \\
& \text { while }\left(k<=\max \text { Iter or } \frac{\sigma_{k}}{\sigma_{k-1}}<\tau_{a}\right) \text {, }
\end{aligned}
$$

where $\eta$ is a non-negative step length which is empirically set to 4 fold of the maximum of magnitude of $\mathbf{A}^{H} \overline{\mathbf{f}}$ in this study. In this study, i.and $h_{\text {lowbound }}$ is the low bound of the threshold parameter for ensuring adequate noise suppression.

Like CORNOL, the algorithm splits the problem of Eq. (6) into two consecutive minimizations that minimize the fidelity and regularization terms separately in each loop. The weight vector is computed before the regularization term is minimized, and it is fixed during minimization of the regularization term. The scale parameter is reduced every few iterations, when the change between results of two consecutive iterations meets a predefined criterion.

\subsection{Numerical simulation}

To verify the feasibility of the proposed mCORNOL, a simulation was performed on a 
Shepp-Logan phantom with a sampling matrix of $256 \times 256$ using an 8 -channel receiving RF coil. The data acquired by each coil were synthetically simulated by multiplying the phantom image with pre-acquired coil sensitivity maps. A non-uniform Fourier transform (NUFFT) [32] was used to generate k-space data. Specifically, the density parameter $\alpha$ [2] in VDS was empirically set to 3, which gave satisfactory results in this study. Further, the VDS trajectory was ameliorated with a regularized slew rate function [33], such that gradient slew rate overflow due to potential singularities of VDS at the k-space center can be avoided. The simulated VDS trajectory has 24 interleaves with 3949 samples for a single interleave. A reduction factor of 5 was used. To test the robustness of these 4 methods, spatially uniform and uncorrelated Gaussian white noise was added to the data.

\subsection{In vivo Experiments}

Healthy volunteers were recruited and provided informed written consent for in vivo studies. T2 weighted brain data were acquired on a Philips 3T Achieva TX system (Philips Healthcare, Best, The Netherlands) with an 8-channel head coil. A spin echo VDS sequence ( $\alpha=3)$ was used with TR/TE of $2500 / 80 \mathrm{~ms}$, FOV of $220 \times 220 \mathrm{~mm}^{2}$, a resolution of $0.86 \times 0.86 \mathrm{~mm}^{2}$ and $5 \mathrm{~mm}$ slice thickness. The VDS acquisition contained 24 arms and the duration for each arm was $16.9 \mathrm{~ms}$. The total acquisition took 60 seconds for 16 axial slices.

An additional set of $\mathrm{T} 2$ weighted data for the knee was acquired on the same scanner using a spin echo VDS sequence $(\alpha=3)$ with an 8-channel knee coil. The sequence had a TR/TE of

$300 / 1.3 \mathrm{~ms}$, FOV of $160 \times 160 \mathrm{~mm}^{2}$, resolution of $0.56 \times 0.56 \mathrm{~mm}^{2}$ and $5 \mathrm{~mm}$ slice thickness. The VDS acquisition contained 36 arms and the duration for each arm was $16.7 \mathrm{~ms}$. 
For both experiments, a spectral pre-saturation inversion recovery scheme was used to suppress fat signal. High order shimming was applied before the data acquisition. Full data sets were first acquired on the scanner, then undersampling with an acceleration factor of 5 was simulated by discarding every other 4 interleaves. Sum-of-squares (SOS) images were used as a gold standard for comparison. The sensitivity maps were estimated using the fully sampled data in the center portion $(8 \times 8)$ of the VDS sampling. These sensitivity maps are used to calculate an intensity map, in order to demodulate the non-uniformity from variation in coil sensitivity in the SOS images.

The undersampled data were reconstructed by 1) un-regularized SENSE, 2) TV regularization, 3) CORNOL, and 4) mCORNOL. Although usually inferior to regularized SENSE for high reduction factors, un-regularized SENSE can provide valuable information on how ill-posed a reconstruction is and what kind of artifacts the ill-posedness introduces. Such information helps identify an ill-posed problem and evaluate regularization methods. For this reason, un-regularized SENSE is used as a basic reference here.

SENSE and TV were implemented in MATLAB (Mathworks Inc., Natick, MA). For CORNOL and mCORNOL, the inner iteration was implemented using $\mathrm{C}$, while the outer iteration is implemented using MATLAB. These four algorithms were compared on a PC with a 3.4GHz Intel Core i7-2600 CPU and $16 \mathrm{~GB}$ of RAM. An implementation of the code and data will be available online.

\subsection{Parameter setup for the reconstruction}

A NUFFT algorithm [32] with Kaiser-Bessel kernel was utilized to grid the non-Cartesian 
data. For NUFFT, the oversampling ratio was 2 and the width of the convolving function was 8 . A Voronoi weight function was utilized to compensate the sampling density of VDS in the k-space domain. In particular, the sampling density of the inner k-space is computed directly using the Voronoi method. For the edge of k-space, a linear extrapolation is used to get the sampling density, based on the sampling density of inner k-space.

The undersampled data were reconstructed by the aforementioned four methods for comparison. To maintain the consistency of numerical implementation, the proximal forward-backward algorithm [31] was used for all methods. Since the patch $p(i)$ in Eq. (4) is an implicit function of the image vector $\overline{\mathbf{u}}$, it is difficult to solve these two unknowns simultaneously. Therefore, in the proximal forward-backward algorithm, the patch is treated as a distinct variable and Eq. (4) is pre-computed at the beginning of each iteration.

For both CORNOL and mCORNOL, the patch size was fixed to $3 \times 3$, and the search window was set to $21 \times 21$ in all experiments, in order to find a balance between computation cost and reconstruction quality. These parameters were investigated in CORNOL [19], and are consistent with most image processing literatures [34,35]. In all experiments, a ROI mask is used to remove the scanned object in the image and the background signal deviation $\sigma_{b}$ is then estimated using the remained signals. The threshold parameter of CORNOL was set to be $\sigma_{b}$. For mCORNOL, the initial threshold parameter $h_{0}$ was empirically set to $h_{0}=5 \sigma_{b}$. In order to ensure adequate noise suppression, the low bound of the threshold parameter was set to $h_{\min }=\frac{1}{5} \sigma_{b}$. The iteration stopping criterion was set to $\tau_{a}=10^{-4}$. The criterion $\tau_{b}$ (in the pseudo code) for scale parameter 
adjustment was set to 0.3 . Generally, about 4 scales were used in the whole reconstruction.

The regularization parameter $\lambda$ was selected from a geometric range based on a root mean square error (RMSE). Although the selected lambda may vary for different image types, this criterion of lambda selection is kept the same for all experiments in study. The RMSE was computed with respect to the fully sampled SOS image. Given that the NUFFT implementation is consistent for all algorithms, RMSE can provide a fair evaluation of different results in presence of data undersampling.

\section{Results}

\subsection{Numerical Simulation}

The convergence behaviors of mCORNOL and un-regularized SENSE for VDS are compared in Fig. 2. For both algorithms, the residual errors decrease quickly in the first few iterations, then begin to rise slowly after reaching the minimum, which is common for non-cartesain iterative reconstruction algorithms [36]. Compared with SENSE, mCORNOL achieves a smaller residual error as well as a faster convergence speed.

The results of the phantom simulations with a reduction factor of 5 are shown in Fig. 3. The SENSE image is degraded by amplified noise (Fig. 3b), which indicates that the SENSE reconstruction is ill-conditioned for the given coil geometry at such a high reduction factor. However, it is worth noting that the spatial distribution of amplified noise is more uniform than Cartesian undersampling cases [19], which results in a better visualization of image structure. The TV image is free of noise, but suffers from artificial intensity variation across the entire image (Fig. 3c). CORNOL preserves image structures better than TV, but there are several 
isolated noisy pixels (indicated by the arrows in Fig. 3d). In contrast, the mCORNOL image shows a much lower level of aforementioned errors (Fig. 3e) than other methods.

In the zoomed-in sections in the $2^{\text {nd }}$ row of Fig. 3 , the SENSE image shows ring-like artifacts which are partially submerged by noise (indicated by the arrow in Fig. 3g). TV suppresses these artifacts, but blurs both low contrast structures and image edges (indicated by the arrowhead and arrow in Fig. 3h, respectively). CORNOL preserves the image structures better, but fails to remove all ring-like artifacts (indicated by the arrows in Fig. 3i). These two phenomena show the conflict between artifact removal and structure preservation, which is difficult to achieve simultaneously by single scale regularization. In the mCORNOL image, the ring artifacts are removed effectively, and the fine structures are also well preserved.

In the difference maps (Fig. $3 \mathrm{k}-\mathrm{n}$ ), the edge blurring effect from TV and residual artifacts from CORNOL can be better visualized, which further confirms that the error level of mCORNOL is the lowest among all four methods.

\subsection{In vivo Experiments}

Fig. 4 shows in vivo brain results with a reduction factor of 5. Similar to the simulation, the SENSE reconstruction introduces intense residual aliasing artifacts (indicated by the arrow in Fig. 4b) and amplified noise. While all three regularization methods successfully remove noise, the residual aliasing artifacts are still visible in the TV and CORNOL images (indicated by the arrows in Fig. 4c and d). But in the mCORNOL image, such aliasing artifacts are reduced to a minimum level.

In the zoomed-in parts, the SENSE image shows a high level of noise as well as residual 
aliasing artifacts (Fig. 4g). Both the shape and scale of the residual aliasing artifacts are close to that of the true image structure, which aggravates the conflict between artifact removal and structure preservation. To resolve the conflict, single scale regularization may have to choose a smoothing scale just between the artifacts and the structures, which is difficult in this situation. For this reason, the TV image exhibits unacceptable structure loss (indicated by the arrow head in Fig. 4h). Meanwhile, there are still slight residual aliasing artifacts in the TV image (indicated by the arrow in Fig. 4h). Similarly, CORNOL better preserves structures, but the residual aliasing artifacts are more obvious (indicated by the arrow in Fig. $4 \mathrm{i}$ and $\mathrm{m}$ ). In contrast, mCORNOL better handles this conflict in the sense that the resultant image is free of both complications (Fig. 4j). This can also be observed clearly in the difference maps in Fig. 4 k-n.

Fig. 5 compares the reconstruction results of the four algorithms for the knee image. For the SENSE image, the noise level is lower than that of the brain image in Fig. $4 b$, but the residual aliasing artifacts are more obvious. TV successfully suppresses the noise. But for residual aliasing artifacts, the TV image doesn't show significant improvement (indicated by the arrow in Fig. 5c). Such residual aliasing artifacts are less in the CORNOL image than those in the TV image, but are still visible (indicated by the arrow in Fig. 5d). In contrast, the mCORNOL image exhibits the lowest level of aliasing artifact in the four methods (Fig. 5e).

Except for noise and aliasing artifacts, the zoomed-in image of SENSE further reveals that the local fine structures are very subtle (indicated by the arrowhead in Fig. $5 \mathrm{~g}$ ). In comparison, the scale of the residual aliasing is quite large (indicated by the arrow in Fig. $5 \mathrm{~g}$ ). This huge difference in scale makes artifact removal and structure preservation incompatible for single 
scale regularization. As a result of this contradiction, both TV and CORNOL image show structure loss and residual aliasing artifacts to certain degree (Fig. 5h and i). The structure loss is less obvious in the CORNOL image than that in the TV image, because CORNOL's prior information matches the image to be solved more closely than TV's. As for mCORNOL, these subtle structures are recovered, which are almost identical to those in the SOS image. Meanwhile, the residual aliasing artifacts are also kept to minimum.

The influence of the different initial threshold parameter $h_{0}$ on mCORNOL is shown in Fig. 6. To reach the minimum residual error, mCORNOL took 54 and 15 iterations for phantom and in vivo images, respectively, with a smaller $h_{0}$ value, while it took 86 and 17 iterations with a larger $h_{0}$ (Fig. 6a and e). However, there are no obvious visual differences between the reconstructed images with different $h_{0}$ values.

Fig. 7 demonstrates the evolution of mCORNOL for the brain data. After first few iterations (5), the image was significantly over-smoothed. As the iteration continued (10-15), detailed image structures were gradually recovered. According to RMSE, the image quality reached its peak at the $15^{\text {th }}$ iteration. When the iteration number (30-90) was further increased, both noise and residual aliasing artifacts returned.

The effects of different regularization parameters $\lambda$ on mCORNOL reconstruction from $10^{-1}$ to $10^{3}$ are studied in Fig. 8. The figure shows the RMSE curve and reconstructed images with underestimated, intermediate and overestimated regularization parameters. The image with underestimated $\lambda$ shows residual aliasing artifacts (Fig. 8b), while the overestimated $\lambda$ shows some flattened areas due to the over-smoothing effect (Fig. 8d). 
The computation time of four methods is given in Table 1. CORNOL and mCORNOL take the longest time, because of the pixel-wise computation of nonlocal operator. This may be reduced if the code is parallelized with more threads. The quantitative evaluation for all experiments is listed in Table 2. For the numerical phantom, the effect of regularization methods is more obvious and mCORNOL shows a more noticeable improvement in terms of RMSE over CORNOL. The underlying reason is believed to be that the noise amplification is more severe than the in vivo experiments. Additionally, the phantom structure is relatively uniform and its edge is clear. This is suitable for TV, CORNOL and mCORNOL. For the in vivo experiments, the noise levels of SENSE are moderate and the effect of regularization methods is not as significant as that of the simulation. In both brain and knee tests, the residual aliasing artifacts are mild which contribute less to the RMSEs. Though mCORNOL can preserve more subtle structures, RMSE differences between CORNOL and mCORNOL are not as obvious as in the simulation. However, regarding absolute values, mCORNOL shows the minimum RMSE among all compared methods.

\section{Discussion}

\subsection{Convergence speed}

The proposed mCORNOL reconstruction shows different convergence speeds in the experiments. For images with sharp edges and large flat areas, such as the Shepp-Logan phantom and knee image, mCORNOL requires more iterations (54 and 58, respectively) to obtain a satisfactory result. A similar phenomenon has been observed with the CORNOL technique as well [19]. The reason may be that the undersampling aliasing artifact of these images also 
contain high contrast and high frequency components, which need more iterations to remove. In comparison, mCORNOL converges faster for the brain image, which has more structures.

\subsection{Nonlocal reconstruction}

LOST (LOw-dimensional-structure Self-learning and Thresholding) is another nonlocal reconstruction method that aims to promote sparsity between nonlocal patches and has received considerable interest and shown remarkable results [22,23]. Unlike mCORNOL, it removes the

exponential function in Eq. (4) and simply uses the patch-based difference $\|p(i)-p(j)\|_{2}^{2}$ for structure detection. This removes any flexibility of scale control for structure estimation. But for retrospective random sampling of Cartesian data, the 3-D sparsity promotion in LOST yields remarkable results $[22,23]$.

In comparison, mCORNOL is tested with in vivo data acquired by VDS on the scanner, which cannot generate aliasing artifacts as incoherent as the retrospective random sampling of Cartesian data that is described in LOST literature. By utilizing the scale-control capacity of Eq. (4), mCORNOL has shown promising performance with less-incoherent aliasing artifacts and other imperfect conditions found in in vivo VDS. Although our method translates well to in vivo imaging, a direction comparison between LOST and mCORNOL for VDS is an important and interesting topic for future work.

\subsection{Successive regularization with varying threshold parameter}

As pointed out previously [37], the successive regularization scheme used in mCORNOL is an effective method to avoid the potential local minima problems in the nonconvex objective function (Eq. (3)). This scheme has also been widely used in L0-norm related nonconvex CS 
methods $[21,38,39]$ with the purpose of approximating L0 metric. In mCORNOL, this scheme focuses on estimating and handling image components with different spatial scales. By utilizing the scale selection ability of Eq. (4), the smoothing constraint scale can be dynamically adjusted throughout the reconstruction process (Fig. 1). As the scale reduces, the number of pixels involved in reconstruction also decreases, reducing the computation burden. Compared with the fixed "semilocal" approach in the previous study [39], mCORNOL may take more computation time in first few iterations, but will use less time in later iterations. Furthermore, this reduction of computation is naturally determined by the estimated structure scale, and thus is more adaptive and flexible.

\subsection{Reconstruction error of $m C O R N O L$}

Since mCORNOL relies on Eq. (4) to detect image structure and impose intra-structure smoothing constraints, the bias in image structure estimation can result in inaccurate smoothing effects and reconstruction errors. As the image structure becomes more complex, the possibility for bias gets higher. Therefore, the in vivo results of mCORNOL showed a higher error level than that of the simulation. Fortunately, by imposing multiscale smoothing constraints, mCORNOL is less prone to the aforementioned bias than the original CORNOL. Except the threshold parameter, the structure estimation parameters in Eq. (4) are the same for both CORNOL and mCORNOL, but the reconstruction error for mCORNOL is smaller than that of CORNOL (Table 1). We also believe that further deblurring may improve the reconstruction accuracy and will be explored in future studies. 


\subsection{Parameter selection consideration}

To remove aliasing artifacts in mCORNOL, the initial threshold $h_{0}$ should optimally be set according to the scale of artifacts. However, in practice it is often difficult to calculate the variance induced by aliasing artifacts. Since the variance $\sigma$ of background signals likely includes contribution from both aliasing artifacts and noise, an initial threshold larger than $\sigma$ should be effective for aliasing artifact removal. Fig. 6 validates that the quality of images reconstructed by mCORNOL is relatively insensitive to the choice of $h_{0}$ if it is sufficiently large. This indicates that mCORNOL is rather stable, although the objective function may be nonconvex with certain weights.

The effect of the regularization parameter on mCORNOL is similar to other regularization methods [14,17-19,21]. The RMSE first decreases to a minimum and then increases, with a small variation range of $10 \%$ (Fig. 8a). Images with intermediate $(\lambda=16.83)$ and overestimated $(\lambda=1022.67)$ regularization parameters also show minor visual differences. This demonstrates that $\mathrm{mCORNOL}$ is relatively insensitive to the regularization parameters.

\section{Conclusions}

A sequential multiscale reconstruction was proposed for fast VDS imaging with a high undersampling factor. It is capable of sequentially performing large-scale artifact suppression and fine-scale structure perseveration by imposing smoothing constraints with gradually reduced scales. This strategy enables a dynamic balance between smoothing constraint and data consistency in an iterative reconstruction process, and alleviates the conflicts between large-scale artifact suppression and fine-scale structure perseveration. The simulation and in vivo studies 
demonstrate the robustness of the proposed method. Compared with other nonlinear methods, mCORNOL may have promising advantages for providing high quality images in parallel imaging.

\section{Acknowledgments}

The authors thank Dr. Condon Lau at the Hong Kong University of Science and Technology for proofreading the manuscript. This work was supported by National Natural Science Foundation of China, Grant No. 11475100 and 61271132.

\section{References}

[1] Tsai CM, Nishimura DG. Reduced aliasing artifacts using variable-density k-space sampling trajectories. Magnetic Resonance in Medicine 2000;43(3):452-458.

[2] Kim D, Adalsteinsson E, Spielman DM. Simple analytic variable density spiral design. Magnetic Resonance in Medicine 2003;50(1):214-219.

[3] Liu C, Bammer R, Kim D, Moseley ME. Self-navigated interleaved spiral (SNAILS): Application to high-resolution diffusion tensor imaging. Magnetic Resonance in Medicine 2004;52(6):1388-1396.

[4] Liu C, Moseley ME, Bammer R. Simultaneous phase correction and SENSE reconstruction for navigated multi-shot DWI with non-cartesian k-space sampling. Magnetic Resonance in Medicine 2005;54(6):1412-1422.

[5] Karampinos DC, Van AT, Olivero WC, Georgiadis JG, Sutton BP. High-resolution diffusion tensor imaging of the human pons with a reduced field-of-view, multishot, variable-density, spiral acquisition at 3 T. Magnetic Resonance in Medicine 2009;62(4):1007-1016.

[6] Deng J, Miller FH, Salem R, Omary RA, Larson AC. Multishot diffusion-weighted PROPELLER magnetic resonance imaging of the abdomen. Invest Radiol 2006;41(10):769-775.

[7] Li TQ, Kim DH, Moseley ME. High-resolution diffusion-weighted imaging with interleaved variable-density spiral acquisitions. Journal of Magnetic Resonance Imaging 2005;21(4):468-475.

[8] Sussman MS, Stainsby JA, Robert N, Merchant N, Wright GA. Variable-density adaptive imaging for high-resolution coronary artery MRI. Magnetic Resonance in Medicine 2002;48(5):753-764.

[9] Liao JR, Pauly JM, Brosnan TJ, Pelc NJ. Reduction of motion artifacts in cine MRI using variable-density spiral trajectories. Magnetic Resonance in Medicine 2005;37(4):569-575.

[10] Kressler B, Spincemaille P, Nguyen TD, Cheng L, Xi Hai Z, Prince MR, Wang Y. Three-dimensional cine imaging using variable-density spiral trajectories and SSFP with application to coronary artery angiography. Magnetic Resonance in Medicine 2007;58(3):535-543. 
[11] Spielman DM, Pauly JM, Meyer CH. Magnetic resonance fluoroscopy using spirals with variable sampling densities. Magnetic Resonance in Medicine 2005;34(3):388-394.

[12] Lee JH, Hargreaves BA, Hu BS, Nishimura DG. Fast 3D imaging using variable-density spiral trajectories with applications to limb perfusion. Magnetic Resonance in Medicine 2003;50(6):1276-1285.

[13] Santos JM, Cunningham CH, Lustig M, Hargreaves BA, Hu BS, Nishimura DG, Pauly JM. Single breath-hold whole-heart MRA using variable-density spirals at 3t. Magnetic Resonance in Medicine 2006;55(2):371-379.

[14] Lustig M, Donoho D, Pauly JM. Sparse MRI: The application of compressed sensing for rapid MR imaging. Magnetic Resonance in Medicine 2007;58(6):1182-1195.

[15] Lustig M, Donoho DL, Santos JM, Pauly JM. Compressed sensing MRI. Signal Processing Magazine, IEEE 2008;25(2):72-82.

[16] Raj A, Singh G, Zabih R, Kressler B, Wang Y, Schuff N, Weiner M. Bayesian parallel imaging with edge-preserving priors. Magnetic Resonance in Medicine 2007;57(1):8-21.

[17] Liu B, King K, Steckner M, Xie J, Sheng J, Ying L. Regularized sensitivity encoding (SENSE) reconstruction using Bregman iterations. Magnetic Resonance in Medicine 2008;61(1):145-152.

[18] Ying L, Liu B., Steckner MC. , Wu Gh, Wu M, Li SJ. A statistical approach to SENSE regularization with arbitrary k-space trajectories. Magnetic Resonance in Medicine 2008;60(2):414-421.

[19] Fang S, Ying K, Zhao L, Cheng J. Coherence regularization for SENSE reconstruction with a nonlocal operator (CORNOL). Magnetic Resonance in Medicine 2010;64(5):1413-1425.

[20] Kai Tobias Block, Martin Uecker, Frahm J. Undersampled radial MRI with multiple coils. Iterative image reconstruction using a total variation constraint. Magnetic Resonance in Medicine 2007;57(6):1086-1098.

[21] Trzasko J, Manduca A. Highly Undersampled Magnetic Resonance Image Reconstruction via Homotopic L0-minimization. Medical Imaging, IEEE Transactions on 2009;28(1):106-121.

[22] Akçakaya M, Basha TA, Goddu B, Goepfert LA, Kissinger KV, Tarokh V, Manning WJ, Nezafat R. Low-dimensional-structure self-learning and thresholding: Regularization beyond compressed sensing for MRI Reconstruction. Magnetic Resonance in Medicine 2011;66(3):756-767.

[23] Ngo TM, Ding H, Akçakaya M, McVeigh ER, Herzka DA. Accelerating T2 Mapping Via Under-Sampled Low-Dimensional-Structure Self-Learning and Thresholding (LOST) Reconstruction. In: Proceedings of the 21th Annual Meeting of ISMRM. Salt lake city, USA; 2013. p 2457.

[24] Wong A, Mishra A, Fieguth P, Clausi D. Sparse Reconstruction of Breast MRI using Homotopic $\mathrm{L}_{0}$ Minimization in a Regional Sparsified Domain. 2010.

[25] Fang S, Wu WC, Guo H. Highly Accelerated Parallel Imaging Using Variable Density Spiral Acquisition and Spatial Adaptive CORNOL Reconstruction. In: Proceedings of the 20th Annual Meeting of ISMRM. Melbourne, Australia; 2012. p 3344.

[26] Wu WC, Fang S, Yuan C, Guo H. High Resolution DTI Using Highly Undersampled Variable Desnity Acquisition and ICORNOL Reconstruction. In: Proceedings of the 20th Annual Meeting of ISMRM. Melbourne, Australia.; 2012. p 1878.

[27] Trzasko J, Manduca A, Borisch E. Sparse MRI reconstruction via multiscale L0-continuation. 2007. IEEE. $p$ 176-180.

[28] Liu B, Abdelsalam E, Sheng J, Ying L. Improved spiral sense reconstruction using a multiscale wavelet model. Proc IEEE ISBI 2008 2008:1505-1508.

[29] Rudin L, Osher S, Fatemi E. Nonlinear total variation based noise removal algorithms. Physica D 
1992;60(1-4):259-268.

[30] Pruessmann KP, Weiger M, Bornert P, Boesiger P. Advances in Sensitivity Encoding With Arbitrary k-Space Trajectories. Magnetic Resonance in Medicine 2001;46(4):638-651.

[31] Combettes PL, Wajs VR. Signal Recovery by Proximal Forward-Backward Splitting. Multiscale Modeling and Simulation 2006;4(4):1168 - 1200.

[32] Fessler JA, Sutton BP. Nonuniform fast Fourier transforms using min-max interpolation. Signal Processing, IEEE Transactions on 2003;51(2):560-574.

[33] Zhao T, Qian Y, Hue Y-K, Ibrahim TS, Boada F, . An Improved Analytical Solution for Variable Density Spiral Design In: Proceedings of the 16th Annual Meeting of ISMRM. Toronoto, Canada.; 2008. p 1342.

[34] Buades A, Coll B, Morel JM. A Review of Image Denoising Algorithms, with a New One. SIAM Multiscale Modeling and Simulation 2005;4(2):490 - 530.

[35] Gilboa G, Osher S. Nonlocal Linear Image Regularization and Supervised Segmentation. SIAM Multiscale Modeling and Simulation 2007;6(2):595 - 632.

[36] Qu P, Zhong K, Zhang B, Wang J, Shen GX. Convergence behavior of iterative SENSE reconstruction with non-Cartesian trajectories. Magnetic Resonance in Medicine 2005;54(4):1040-1045.

[37] Chartrand R. Fast algorithms for nonconvex compressive sensing: MRI reconstruction from very few data. 2009. IEEE. p 262-265.

[38] Mohimani H, Babaie-Zadeh M, Jutten C. A Fast Approach for Overcomplete Sparse Decomposition Based on Smoothed $<$ formula formulatype $=$. Signal Processing, IEEE Transactions on 2009;57(1):289-301.

[39] Wong A, Mishra A, Fieguth P, Clausi D. Sparse Reconstruction of Breast MRI using Homotopic L0 Minimization in a Regional Sparsified Domain. 2010. 
Table 1. Computation time of un-regularized SENSE, TV, CORNOL, and mCORNOL (unit: second).

\begin{tabular}{ccccc}
\hline data set & SENSE & TV & CORNOL & mCORNOL \\
\hline phantom & 11.52 & 37.52 & 56.30 & 93.92 \\
brain & 5.29 & 12.26 & 30.89 & 51.17 \\
knee & 50.05 & 188.51 & 465.95 & 476.02 \\
\hline
\end{tabular}

Table 2. RMSE of images reconstructed by un-regularized SENSE, TV, CORNOL, and mCORNOL.

\begin{tabular}{ccccc}
\hline data set & SENSE & TV & CORNOL & mCORNOL \\
\hline phantom & 17.17 & 2.28 & 1.85 & 0.96 \\
brain & 15.07 & 7.96 & 6.53 & 5.56 \\
knee & 6.36 & 4.06 & 3.10 & 2.32 \\
\hline
\end{tabular}




\section{Figure Caption}

FIG. 1. The scale selection effect of nonlocal operators. The top row compares the shapes of the exponential term in Eq. (4) with different threshold parameters. The bottom row shows the spatial distribution of the corresponding weights calculated using the threshold parameters in the top row.

FIG. 2. Convergence behavior of SENSE and mCORNOL ford VDS. A reduction factor of 5 was used in the simulation.

FIG. 3. Numerical simulation for a reduction factor of 5 with synthetically added Gaussian white noise. The first row shows the full size images from (a) the original phantom; (b) SENSE; (c) TV; (d) CORNOL; and (e) mCORNOL. The second row shows corresponding zoomed-in parts and the third row shows the corresponding difference maps (scaled by a factor of 2.5 for better visualization).

FIG. 4. Reconstruction results of the brain data with a reduction factor of 5.The first row shows the full size images from (a) sum-of-squares; (b) SENSE; (c) TV; (d) CORNOL; and (e) mCORNOL. The second row shows corresponding zoomed-in parts and the third row shows corresponding difference maps (scaled by a factor of 5 for better visualization).

FIG. 5. Reconstruction results of knee data with a reduction factor of 5. The first row shows the 
full size images from (a) sum-of-squares; (b) SENSE; (c) TV; (d) CORNOL; and (e) mCORNOL. The second row shows corresponding zoomed-in parts, and the third row shows corresponding difference maps (scaled by a factor of 3 for better visualization). The arrows in the first and second rows point out the residual aliasing artifacts in SENSE, TV and CORNOL images, respectively. The arrowheads in the second row show the structure blurring effect in SENSE, TV and CORNOL images, respectively.

FIG. 6. The influence of initial threshold $h_{0}$ on the convergence behaivor and reconstruction results of mCORNOL. (a) convergence behavior of mCORNOL with different $h_{0}$ for phantom; (b) phantom results with a small $h_{0}$; (c) phantom reconstruction results with a large $h_{0}$; (d) the difference between (b) and (c), which was scaled 10 times for better visualization; (e) convergence behavior of mCORNOL with different $h_{0}$ for brain imaging; (f) brain results with a small $h_{0} ;(\mathrm{g})$ brain reconstruction result with a large $h_{0} ;(\mathrm{h})$ the difference between $(\mathrm{f})$ and $(\mathrm{g})$, which was scaled by a factor of 10 .

FIG. 7. Brain images with a reduction factor of 5 with different iterations $(5,10,15,3060$, and 90).

FIG. 8. The influence of regularization parameter $(\lambda)$ on the mCORNOL reconstruction. (a) The RMSE plot of mCORNOL reconstruction against the logarithm of regularization parameters; (b) reconstruction with an underestimated regularization parameter; (c) reconstruction with an 
optimal regularization parameter; (d) reconstruction result with an overestimated regularization parameter. 